\title{
Determination of Fiber Contents in Blended Textiles by NIR Combined with BP Neural Network
}

\author{
Li Liu, Li Yan, Yaocheng Xie, and Jie Xu \\ School of Material Science and Engineering, Wuhan Textile University, Wuhan 430073, China \\ Correspondence should be addressed to Li Liu; li_liu2006@yahoo.com.cn
}

Received 22 March 2013; Accepted 20 April 2013

Academic Editors: I. Frydrych, A. A. Merati, and C. H. Park

Copyright (C) 2013 Li Liu et al. This is an open access article distributed under the Creative Commons Attribution License, which permits unrestricted use, distribution, and reproduction in any medium, provided the original work is properly cited.

Fiber contents in cotton/terylene and cotton/wool blended textiles were tested by near infrared (NIR) spectroscopy combined with back propagation (BP) neural network. Near infrared spectra of samples were obtained in the range of $4000 \mathrm{~cm}^{-1} \sim 10000 \mathrm{~cm}^{-1}$. Wavelet Transform (WT) was used for noise reduction and compression of spectra data. The correction models of cotton/terylene and cotton/wool contents based on BP neural network and reconstructed spectral signals were established. The number of hidden neurons, learning rate, momentum factor, and learning times was optimized, and decomposition scale of WT was discussed. Experimental results have shown that this approach by Fourier transformation NIR based on the BP neural network to predict the fiber content of textile can satisfy the requirement of quantitative analysis and is also suitable for other fiber content measurements of blended textiles.

\section{Introduction}

Textiles are necessaries of human life. With the development of textile industry and the people's living standards, the kinds of pure textile, blended textile, and intertexture are increasing gradually. The requirement of analysis to fibers is also raised in the fields of production, scientific research, and trade. The various fiber content is index of textile quality, and how to measure it has an important meaning. Traditional chemical solution is a quantitative detecting method, which has long testing time, and a series of solvents should be prepared to dissolve the fiber, and lots of harmful gases would be produced and pollute the working environment [1].

NIR is referred to as the electromagnetic wave in the wavelength range of $780 \mathrm{~nm} \sim 2526 \mathrm{~nm}$, which has wide spectrum bands, weak absorption, and more information and is produced by the absorption of frequency doubling and frequency summing caused by molecular radicals' vibration. Using stoichiometry method to solve the extraction of spectrum information and background interference, the good testing results will be obtained. Combination of NIR with stechiometry is more suitable to quantitive analysis and has been already applied to the fields of medicine, food, agriculture, chemical industry, and environment monitoring
[2-8]. Applications of NIR to testing the fiber contents in textiles have been reported [9-12]. Most of these reports used the pretreatment method to preprocess spectrum data and sieve method to screening variables and then built up the model of partial least square (PLS) method. The progression is inconvenient.

In our previous work, the fiber contents of cotton/terylene blended textiles were predicted by NIR combined with BP neural network, and a decomposition scale 3 of WT was used. In this work, the near infrared spectra of 51 samples with different cotton/terylene and cotton/wool contents were directly reduced and denoised by Wavelet Transform (WT). The correction models of cotton/terylene and cotton/wool contents in blended textiles based on BP neural network were established. The number of hidden neurons, learning rate, momentum factor, and learning times was optimized, and a different decomposition scale of WT was used. Comparison of prediction results of BP models with PLS model was given.

\section{Materials and Methods}

2.1. Materials Preparation. The materials were provided by School of Materials Science and Engineering, Wuhan University of Science and Engineering. The textiles of pure 


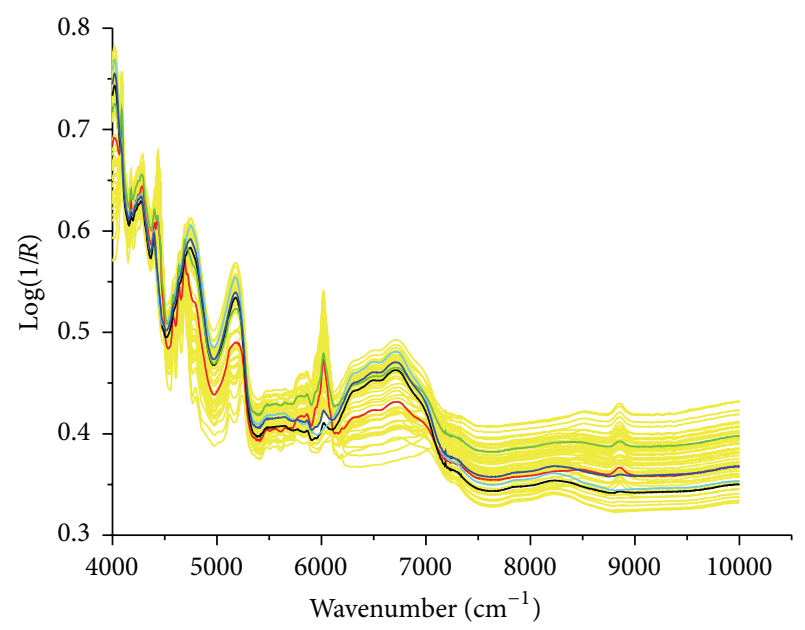

FIGURE 1: NIR of cotton and terylene textile mixture.

cotton and pure terylene or wool were crushed into fibers about $1 \mathrm{~mm}$ long, respectively, and fully mixed with different ratio in order to prepare the blended samples with different cotton/terylene or cotton/wool contents. Each sample had the same mass of $0.05 \mathrm{~g}$, and 51 samples were prepared, in which the content of cotton increased by mass fraction gradient of $2 \%$, and the content of terylene or wool decreased by mass fraction gradient of $2 \% .10$ samples were selected randomly as validation set, and the other 41 samples were used as calibration set.

2.2. NIR Collection. Before NIR collection, the samples were dried at the temperature of $60^{\circ} \mathrm{C}$ by the galvanothermy blasting dry oven with constant temperature made by Shanghai Suopu Instrument Co., Ltd., whose type is DHG-9030A.

We used the Fourier NIR analyzer with the type of ANTARIS II, made by Thermo Nicolet Instrument Inc. of USA. The detector was InGaAs detector, and the scanning wavenumber range was from $4000 \mathrm{~cm}^{-1}$ to $10000 \mathrm{~cm}^{-1}$. Accessories of integrating sphere for diffuse reflection were adopted. The sampling resolution was $8 \mathrm{~cm}^{-1}$. The light source was a halogen tungsten lamp. The sample cell was rotated $120^{\circ}$ every time. For each sample, the spectrum data were acquired at three angles, and the average was used. All the spectral data were collected under the condition of constant temperature of $20^{\circ} \mathrm{C}$. The NIR of samples with different cotton/terylene contents was shown in Figure 1.

2.3. BP Neural Network. Artificial neural network (ANN) has a powerful learning ability and can realize the high nonlinear mapping between the input and output [13]. BP neural network is a study process of error back propagation arithmetic which includes two processes of information forward propagation and error back propagation. The neurons of the input layer receive the external information and pass it to neurons of intermediate layers. The intermediate layer can be designed as single or multihidden configuration. A forward learning propagation is finished when the information passed to neurons of the output layer by the last hidden layer is further treated, and the results are outputted to the outside by the output layer. The stage of error back propagation will begin when the factual output does not accord with the expected output. The error is back propagated from the output layer to the hidden layer and the input layer and corrects the weights of each layer according to gradient descent. The circular information of forward propagation and error back propagation is a course that the weight of each layer is adjusted continually and the neural network is trained constantly. This course will not be stopped until the output error is reduced to the accepted degree, or the preset times of training are reached.

2.4. Wavelet Transform. Wavelet analysis is a new field of mathematics, which has wide application such as smoothing, noise reduction, compression, and transfer. Compared with linear smoothing, the WT smoothing has a distinct advantage especially on details of signal [14]. The original data were denoised by WT. Two functions of ddencmp and wdencmp were used. Ddencmp function can create the threshold scheme of noise reduction by WT, that is, find the default. Wdencmp function can use WT to denoise. The main procedure to compress one-dimensional signal includes the wavelet analyzing of signal, the threshold treatment for high frequency coefficient and one dimension wavelet reconstruction. The two WT functions of wavedec and appcoef provided by MATLAB were used to compress the spectrum data and accelerate the model calculation, where wavedec is one-dimensional wavelet analyzing function of multiple scales, and appcoef is a picking-up function of onedimensional wavelet low frequency coefficient.

\section{Results and Discussion}

3.1. Spectral Band Selection. For each sample, NIR included 601 spectrum data. If these 601 spectral data were all used, the dimension of input data would be very large and result in low speed of computer treatment. From Figure 1, we could find that the characteristic absorption peaks were focused in the wavenumber range from $4000 \mathrm{~cm}^{-1}$ to $7000 \mathrm{~cm}^{-1}$, which included lots of information about samples. So the spectral data in this wavenumber range were used as spectrum analysis. The number of spectral data in the wavenumber range of $4000 \mathrm{~cm}^{-1} \sim 7000 \mathrm{~cm}^{-1}$ was 321 .

3.2. Wavelet Compression of Spectrum Data and the Choice of Wavelet Modulus. When using wavelet to compress the data, the more the decomposition scale is, the fewer the data size and the dimension of input data are, which hoists the prediction speed of correction model. At the same time the severe distortion leads to the low prediction accuracy of correction model. Taking into account both the prediction speed and accuracy of correction model, the decomposition scales of 3, 4, and 5 were selected, respectively, and wavelet basis function was bd1. The NIR models of blended textile were built up, and the spectrum data of 321 were compressed to 41,21 , and 11 . 
TABLE 1: Prediction results of validation samples at decomposition scale of 3 .

\begin{tabular}{lccccc}
\hline Serial number of samples & \multicolumn{3}{c}{ Cotton } & \multicolumn{3}{c}{ Terylene } \\
PV (\%)
\end{tabular}

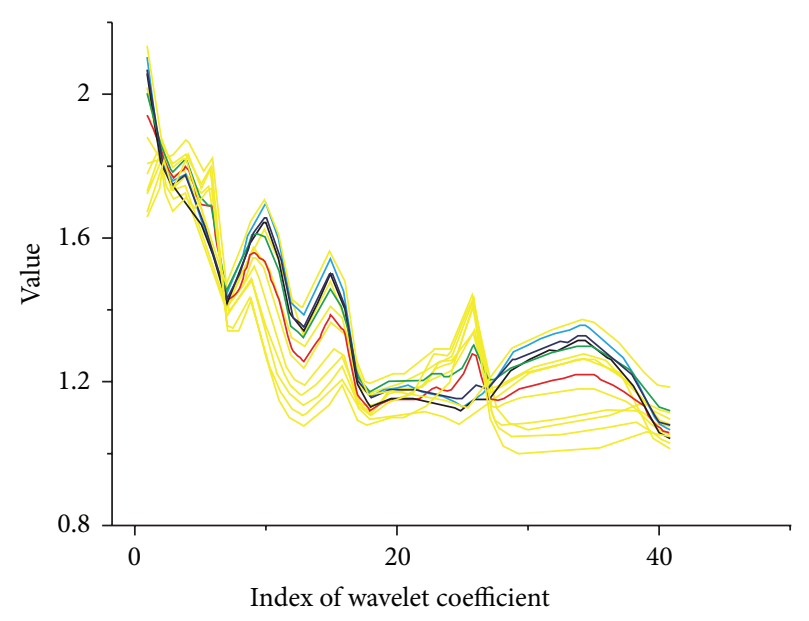

Figure 2: Reconstructed spectral signal.

The reconstructed spectrum signals at decomposition scale of 3 are shown in Figure 2. Comparing Figure 2 with NIR of blended textiles, it can be seen that the reconstructed spectrum signals with wavelet modulus of 41 kept the original information of characteristic peaks in large degree and could not affect the prediction accuracy of correction model.

\subsection{Optimization of Network Parameters}

3.3.1. Determination of Number of Neurons in Hidden Layers. The absorbency in wavenumber range of $4000 \mathrm{~cm}^{-1} \sim$ $7000 \mathrm{~cm}^{-1}$ was the input of neural network. With the wavelet analyzing scale of 3 , the number of nodes of input layer was 41 , and the number of nodes of output layer was 2, corresponding to the contents of cotton and terylene. The number of hidden nodes determined the complexity of BP neural network. If the number of neurons in output layer was extensively few, the error would vary little after it decreased to a certain extent, the weights of BP neural network would not be enough, and good prediction results could not be achieved. If the number of neurons in output layer was too much, the network structure would be too complex, the training time would be very long and be difficult to converge, and the phenomena of overfitting, oscillation would appear. In our work, the number of hidden nodes was directly decided by the prediction results.

The number of hidden neurons was selected in the range of 15 22; the prediction effect for cotton/terylene samples was checked according to root-mean-square error (RMSE) and the mean absolute error (MAE). The fewer the RMSE and MAE were, the higher the precision of calibration and prediction was. For the wavelet decomposition scale of 3, when the number of hidden neurons was selected as 17 , the predicting precision was higher. For the wavelet decomposition scale of 4 , the structure of network was 2113-2. For the wavelet analyzing scale of 5, the structure of network was 11-9-2.

3.3.2. Choice of Training Parameters of Network. The training times and the predicting ability of network were the main evaluating indicators of ANN. It was expected that the reliable prediction results for unknown samples were provided, and the training times were not too much. Therefore, for the 51 samples, 10 samples were selected randomly as validation set, and the other 41 samples were used as training set. Learning rate had much effect on iteration, and its value was selected in $0.01 \sim 0.8$ commonly. Large learning rate might lead to instability of network, and little learning rate could lead to long training time and low convergence speed. For raising the convergence speed, the momentum item was improved. By debugging, the training parameters of network were made certain: the learning rate was 0.6 , the momentum factor was 0.3 , target error value was set as 0.00003 , and the training times were set as 5000 .

3.4. Results and Analysis. Table 1 gives the prediction results of cotton/terylene validation samples at decomposition scale of 3, where CV, PV, AE, MAE, RMSE, and $R_{P}$ are the chemical value, prediction value, absolute error, mean absolute value, root-mean-square error, and correlation coefficients respectively. Table 2 gives the comparison of prediction results of BP model at analyzing scales of 3, 4, and 5 (marked as WTca3-BP, WT-ca4-BP, and WT-ca5-BP correspondingly) with PLS model. By using WT-ca3-BP model, the AE was less than $4 \%$, the MAE was $1.26 \%$ for cotton and $1.29 \%$ for terylene, 
TABLE 2: Comparison of prediction results of BP models with PLS model for cotton and terylene blended textile.

\begin{tabular}{|c|c|c|c|c|c|c|}
\hline \multirow{2}{*}{ Model } & \multicolumn{3}{|c|}{ Cotton } & \multicolumn{3}{|c|}{ Terylene } \\
\hline & MAE (\%) & RMSE (\%) & $R_{P}$ & MAE (\%) & RMSE (\%) & $R_{P}$ \\
\hline WT-ca3-BP & 1.26 & 1.86 & 0.998 & 1.29 & 1.89 & 0.998 \\
\hline WT-ca4-BP & 2.16 & 2.66 & 0.997 & 2.42 & 2.91 & 0.996 \\
\hline WT-ca5-BP & 3.19 & 3.74 & 0.995 & 3.22 & 3.75 & 0.995 \\
\hline PLS & 4.73 & 5.99 & 0.981 & 4.68 & 7.03 & 0.980 \\
\hline
\end{tabular}

TABLE 3: Test results of cotton and wool mixture validation samples.

\begin{tabular}{|c|c|c|c|c|c|c|}
\hline \multirow{2}{*}{ Serial number of samples } & \multicolumn{3}{|c|}{ Cotton } & \multicolumn{3}{|c|}{ Wool } \\
\hline & $\mathrm{CV}(\%)$ & PV (\%) & $\mathrm{AE}(\%)$ & CV (\%) & PV (\%) & $\mathrm{AE}(\%)$ \\
\hline 5 & 8.00 & 7.68 & 0.32 & 92.00 & 91.61 & 0.39 \\
\hline 8 & 14.00 & 12.11 & 1.89 & 86.00 & 87.11 & -1.11 \\
\hline 14 & 26.00 & 27.94 & -1.94 & 74.00 & 71.60 & 2.40 \\
\hline 17 & 32.00 & 31.94 & 0.06 & 68.00 & 67.78 & 0.23 \\
\hline 25 & 48.00 & 46.60 & 1.40 & 52.00 & 53.74 & -1.74 \\
\hline 29 & 56.00 & 60.25 & -4.25 & 44.00 & 40.32 & 3.68 \\
\hline 33 & 64.00 & 62.01 & 1.99 & 36.00 & 38.39 & -2.39 \\
\hline 36 & 70.00 & 72.28 & -2.28 & 30.00 & 27.22 & 2.78 \\
\hline 42 & 82.00 & 84.54 & -2.54 & 18.00 & 14.37 & 3.63 \\
\hline 47 & 92.00 & 92.66 & -0.66 & 8.00 & 6.19 & 1.81 \\
\hline MAE (\%) & & 1.73 & & & 2.01 & \\
\hline RMSE (\%) & & 2.20 & & & 2.44 & \\
\hline
\end{tabular}

and the RMSE was $1.86 \%$ for cotton and $1.89 \%$ for terylene. Correlation coefficients $\left(R_{P}\right)$ of cotton and terylene contents between prediction value and chemical value both were 0.998, and the prediction precision was the best. Comparing the BP models with PLS model, it can be found that the prediction precisions at the wavelet analyzing scales of 3 , 4 , and 5 both were better than PLS model. Therefore BP model was more suitable for determination of fiber contents of blended textile than PLS model. For cotton/wool samples, the best prediction accuracy was achieved when the compression scale was 4 and the network configuration was 2113-2. RMSE was $2.20 \%$ and $2.44 \%$, respectively (shown in Table 3).

\section{Conclusions}

A method for quantitative measurement of contents of blended textile by NIR based on BP neural network was investigated. 51 samples were prepared, in which 10 samples were selected randomly as validation set and the other 41 samples were used as calibration set. WT was used for the noise reduction and compression of spectra data. The correction models of cotton/terylene and cotton/wool contents based on BP neural network were established, the training parameters of network were optimized, and their prediction results were compared with PLS model. The BP model combined with WT has the characteristics of simplicity, and stability, high prediction accuracy and is fit for quantitative analysis for textile fiber.

\section{Acknowledgments}

This work was supported in part by the National Natural Science Foundation of China no. 6110803 and no. 51003082 and Natural Science Foundation of Hubei Province no. 2009 CBD295.

\section{References}

[1] Y. Zhou, H. Xu, and Y. Ying, "NIR analysis of textile natural raw material," Spectroscopy \& Spectral Analysis, vol. 28, no. 12, pp. 2804-2807, 2008.

[2] H. Wang, W. Li, and Y. Liu, "Study on discrimination of varieties of corn using near-infrared spectroscopy based on GA and LDA," Spectroscopy \& Spectral Analysis, vol. 31, no. 3, pp. 669672, 2011.

[3] D. Sun, Y. Li, Q. Wang, and C. Le, "Remote sensing retrieval of CDOM concentration in Lake Taihu with hyper-spectral data and neural network model," Geomatics \& Information Science of Wuhan University, vol. 34, no. 7, pp. 851-855, 2009.

[4] T. A. Lestander and P. Geladi, "NIR spectral information used to predict water content of pine seeds from multivariate calibration," Canadian Journal of Forest Research, vol. 35, no. 5, pp. 1139-1148, 2005.

[5] V. A. Saptari, K. Youcef-Toumi, and J. Zhang, "NIR measurements of glucose in synthetic biological solutions using highthroughput angle-tuned filter spectrometer," in Proceedings of the 4th SPIE Optical Diagnostics and Sensing, vol. 5325, pp. 1-10, January 2004.

[6] Y. Shan, X. R. Zhu, Q. S. Xu, and Y. Z. Liang, "Determining the contents of fat and protein in milk powder by using near 
infrared spectroscopycombined with wavelet transform and radical basis function neural networks," Journal of Infrared \& Millimeter Waves, vol. 29, no. 2, pp. 128-131, 2010.

[7] W. Liu, Q. R. Chang, M. Guo, D. X. Xing, and Y. S. Yuan, "Detection of leaf nitrogen content of summer corn using visible/near infrared spectra," Journal of Infrared \& Millimeter Waves, vol. 30, no. 1, pp. 48-54, 2011.

[8] Y. Zhang, L. D. Zhang, Q. L. Bai, and S. J. Chen, "Nondestructive identification of different oil content maize kernels by nearinfrared spectra," Spectroscopy \& Spectral Analysis, vol. 29, no. 3, pp. 686-689, 2009.

[9] G. F. Wu, D. S. Zhu, and Y. He, "Identification of fine wool and cashmere by using Vis/NIR spectroscopy technology," Spectroscopy and Spectral Analysis, vol. 28, no. 6, pp. 1260-1263, 2008.

[10] B. Chen, G. Cui, S. Z. Jin, and X. T. Wang, "Application of near infrared spectra in rapid inspection of cotton contents," Journal of Jiangsu University, vol. 28, no. 3, pp. 185-188, 2007.

[11] H. N. Feng, B. Gan, and S. Z. Jin, "Concentration test of textile mixture of cotton and terlene with NIR method," Laser \& Infrared, vol. 35, p. 768, 2005.

[12] B. Chen, X. T. Wu, and K. Ni, "Application of correlation analysis in rapid detecting silk content of fabric textile by NIR," Spectroscopy Instrument and Analysis Z, vol. 1, p. 52, 2006.

[13] Z. X. Ge and Z. Q. Sun, Eds., The Theory of Artificial Neural Network and Its Realization in MATLAB7, Electronics Industry Press, Beijing, China, 2007.

[14] Center for the Research and Development of FeiSi Technology, Ed., The Theory of Wavelet Analysis and Its Realization in MATLAB7, Electronics Industry Press, Beijing, China, 2005. 

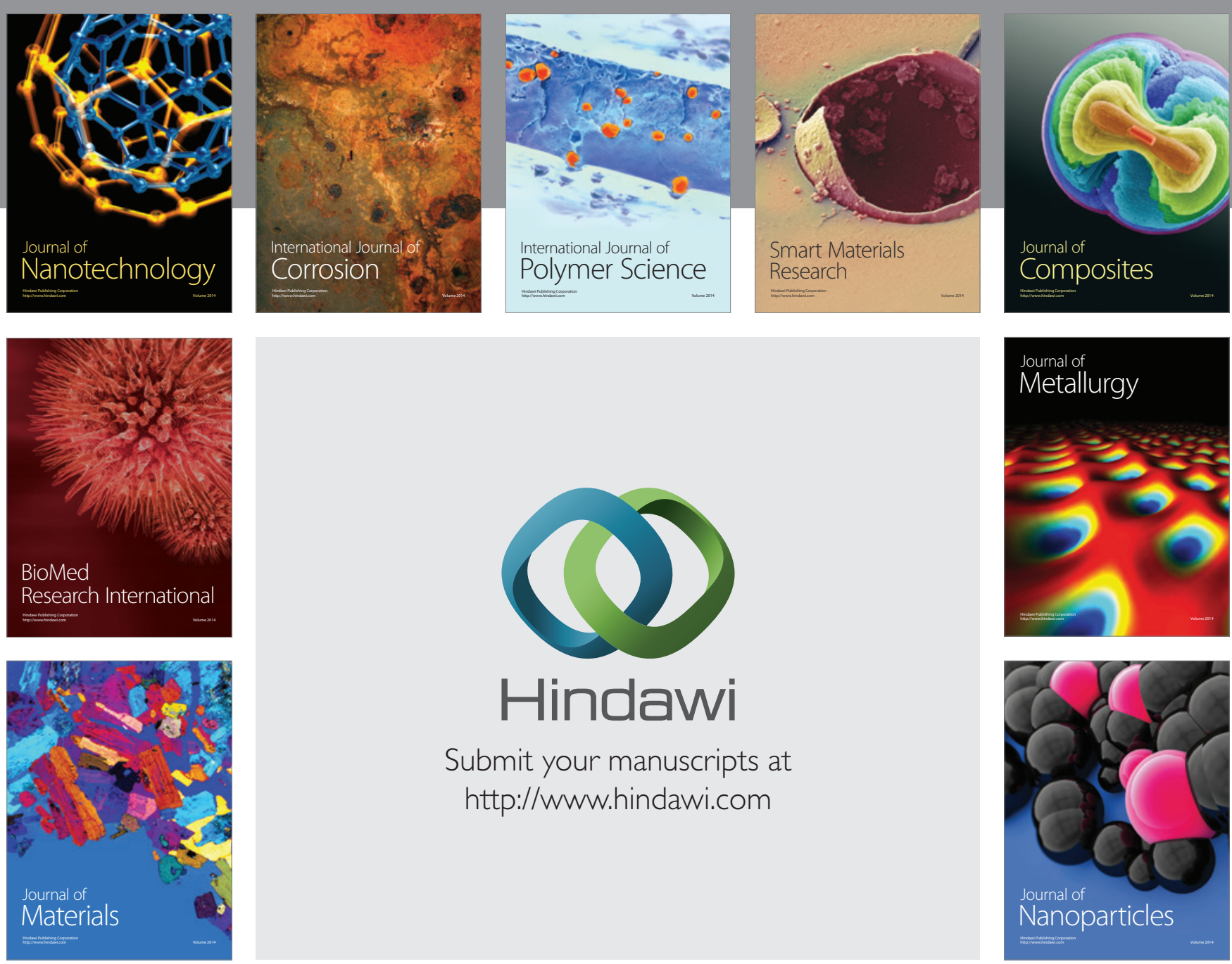

Submit your manuscripts at http://www.hindawi.com
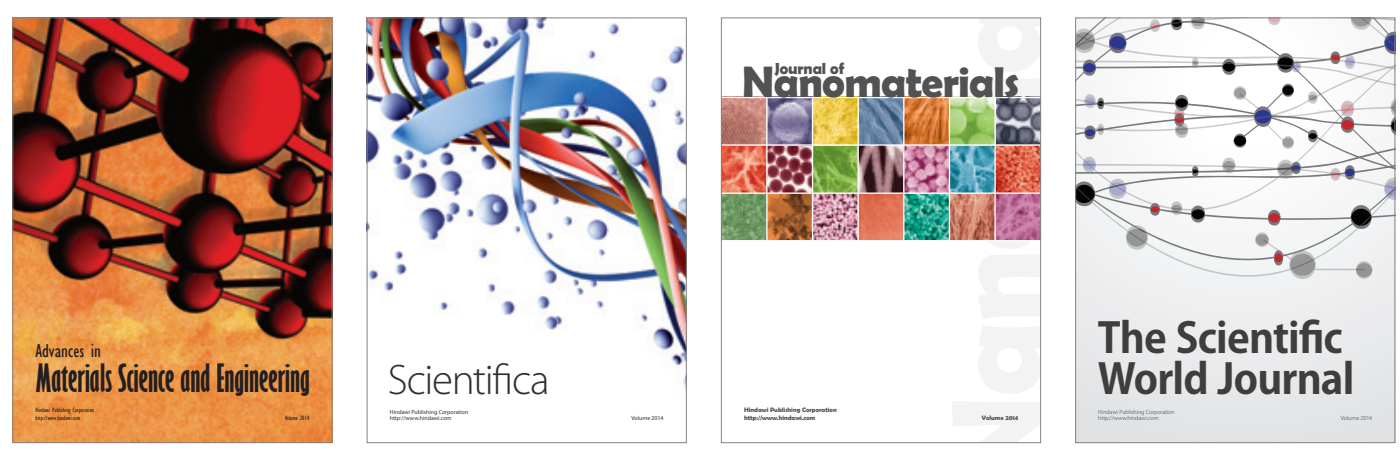

\section{The Scientific World Journal}
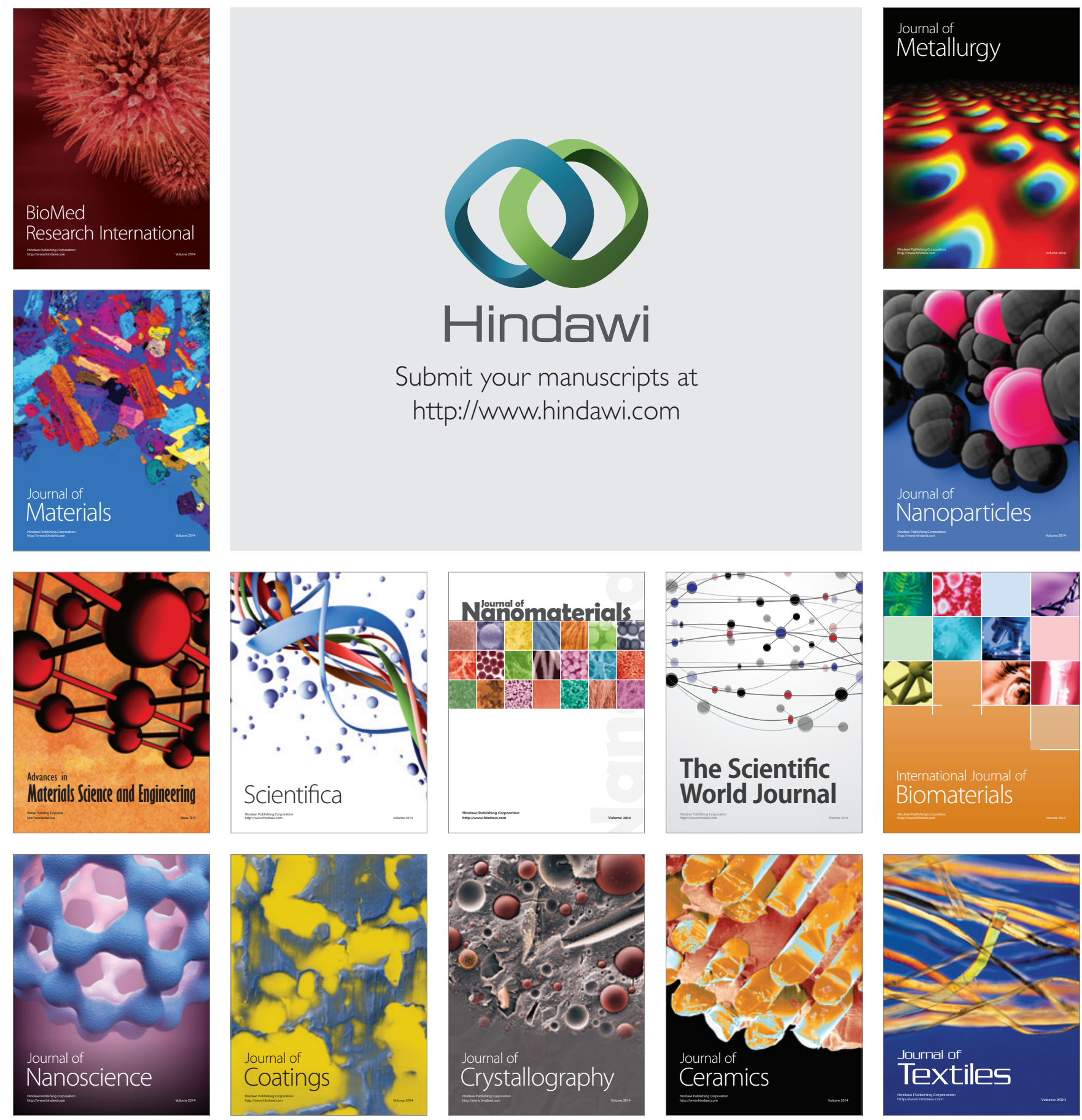